\title{
A CORRELATION BETWEEN FASTING BLOOD GLUCOSE AND PERIODONTAL FINDING IN DIABETIC PATIENTS HAVING CHRONIC PERIODONTITIS AND TREATED WITH (TRAUMEEL) DRUG
}

\author{
Maha R. Taalab*
}

\begin{abstract}
Objective to investigate the influence of Traumeel drug as adjunctive to non surgical periodontal intervention in patients with poorly controlled type 2 diabetes associated with chronic periodontitis on both periodontal parameters and metabolic findings, and to correlate the levels of fasting blood glucose (FBG), and fasting blood insulin (FBI) to the clinical periodontal parameters in those patients after treatment.
\end{abstract}

Methods and Patients A total of 20 patients having moderately poorly controlled (HbA1c between $8 \%-10 \%$ ) type 2 diabetes mellitus associated with moderate to severe chronic periodontitis were selected and randomly divided into two groups according to whether they underwent periodontal intervention alone (control group) or periodontal intervention adjunctive to traumeel tablets with a dose of of 1 tablet 3 times daily for 1 week. The levels of serum tumor necrosis factor á (TNF- $\alpha$ ), FBG,FBI and glycosylated hemoglobin $(\mathrm{HbC} 1)$ were measured at baseline and after 3 months. The periodontal parameters including Probing pocket depth(PPD). Clinical attachment loss (CAL) and Modified Gingival index (MGI) were also measured at baseline and after 3 months

Results The levels of both metabolic parameters and clinical periodontal parameters were improved significantly in both test and control groups after 3 months but on comparing percent of change D at $\mathrm{p} \leq 0.05$ between control and tablet groups after 3 months ,test group showed more reduction in all parameters except Hbc1compared to control group .There were also a statistically significant correlation between periodontal parameters and FBG and FBI in the test group only.

Conclusion The adjunctive use of homeopathic Traumeel tablets with periodontal intervention offered better results concerning periodontal and metabolic state .

KEY WORDS: Type 2 diabetes mellitus, chronic periodontitis, FBI, FBG, and Hbc1.

\section{INTRODUCTION}

Periodontitis is a common chronic inflammatory disease, caused by gram-negative infection and characterized by periodontal pocket formation, loss of connective tissue attachment and alveolar bone resorption which can result in tooth mobility.(1)

\footnotetext{
* Lecturer of Periodontology, Oral Medicine, Diagnosis and Radiology, Faculty of Dentistry, Alexandria University
} 
Diabetes mellitus (DM) is a clinically and genetically heterogeneous group of metabolic disorders manifested by abnormally high levels of glucose in the blood. The hyperglycemia is the result of a deficiency of insulin secretion caused by pancreatic $\beta$-cell dysfunction or resistance to the action of insulin in peripheral tissue or a combination. (2)

DM is either type 1 or type 2 , type 2 diabetes was known previously as non insulin-dependent diabetes. It is now known that type 2 diabetic patients have insulin resistance which is considered to play central role in its pathogenesis, while autoimmune destruction of $\beta$ cells does not occur, and patients retain the capacity for some insulin production. ${ }^{(3)}$

Authors have reported a bidirectional relationship between the mechanism of type 2 diabetes and periodontitis as not only DM adversely affects periodontal condition but also periodontitis adversely influences glycemic control, increasing the risk of complications in diabetic Patients. ${ }^{(4)}$

Several findings indicate that chronic low-grade inflammation is closely involved not only in the pathogenesis of type $2 \mathrm{DM}$ and its complications, ${ }^{(5)}$ but also in the pathogenesis of periodontal diseases, whereby cytokines play a central role in the host's response to the periodontal biofilm. ${ }^{(5-7)}$

Evidence suggests that Tumor necrosis factor-alpha (TNF- $\alpha$ ) has been implicated as a causative factor in pathogenesis of type 2 diabetes. Thus, current evidence suggests that administration of exogenous TNF- $\alpha$ to animals can induce insulin resistance, whereas neutralization of TNF- $\alpha$ can improve insulin sensitivity. ${ }^{(8)}$

It was found also that (TNF- $\alpha$ ) is a major mediator of the immune-inflammatory response and play an important role in the pathogenesis and progression of chronic periodontal disease (PD) ${ }^{(6)}$

On the other hand free radicals are considered to be a common factor in the pathogenesis of both periodontitis and diabetes mellitus. Bacteria implicated in the etiology of periodontitis causes destruction of connective tissue and bone which occur through stimulation of polymorphonuclear neutrophils (PMN). Polymorphonuclear neutrophils produces free radicals via respiratory burst as a part of host response to infection. These free radicals damage proteins, lipids, carbohydrates and nucleotides in the tissues. $^{(9-12)}$

Free radicals may also play an important role in the causation and complications of DM. (10-13) $^{(1)}$ In DM, alterations in the endogenous free radical scavenging defense mechanisms may result in oxidative damage and severe tissue injury. ${ }^{(11-13)}$

Homeopathy is a form of alternative therapy which comes from the Greek words homeo, meaning similar, and pathos, meaning suffering or disease. Homeopathy seeks to stimulate the body's ability to heal itself by giving very small doses of highly diluted natural substances. The principle of dilutions (or "law of minimum dose") states that the lower the dose of the medication, the greater its effectiveness. ${ }^{(14)}$

Traumeel, is a homeopathic remedy that is made of a mixture of 12 botanical and two mineral substances in micro- or ultra low-dilutions. Traumeel results in rapid reduction of inflammation and healing promotion. It has also a pronounced analgesic, antiedematous effects. Thus it is considered to be a potent anti-Inflammatory. ${ }^{(15)}$

Mode of action of Traumeel was proved in various clinical applications which suggests that Traumeel works in a different way compared to non steroid anti-inflammatory drugs (NSAIDs). Traumeel and its constituents exert bioregulatory effects via the inhibition of various proinflammatory cytokines, such as interleukin IL-2, IL-6, and tumor necrosis factor-alpha (TNF- $\alpha$ ) .It can also perform modulation of regulatory cells/transforming growth factorbeta (TGF- $\beta$ ); and inhibition of IL- 8 as proved with Ludmila, etal 2004 who used Traumeel in the management of patients with chronic periodontitis and reached an excellent results.$^{(16)}$ 
More over Traumeel was proved to be an antioxidant as it can significantly reduce production of superoxide anion by peripheral blood polymorphonuclear neutrophils of periodontitis patients. ${ }^{(17)}$

Thus as both oxidative stress and inflammatory cytokines can worsen the glycemic control of diabetic patients therefore decreasing them by traumeel drug can improve the glycemic control of those patients.Also evidence suggests that periodontal therapy can decrease the intraoral bacterial bioburden, periodontal inflammation, and inflammatory cytokines, and so can improve glycemic control so the study hypothesis is about the positive impact of adding traumeel drug as an antioxidant and anti-inflammatory drug to the treatment protocol for type 2 DM having chronic periodontitis.. ${ }^{(18)}$

\section{Objectives:}

This study attempts to investigate the influence of Traumeel drug as adjunctive to non surgical periodontal intervention in patients with poorly controlled type 2 diabetes associated with chronic periodontitis on both periodontal parameters and metabolic findings, and to correlate the levels of FBG and FBI to the clinical periodontal parameters in those patients after treatment.

\section{MATERIALS AND METHODS}

\section{Study design}

The study design is controlled clinical trial so a total of 20 patients having type 2 diabetes mellitus associated with chronic periodontitis were selected from the oral medicine and periodontology department, faculty of dentistry, Alexandria University.

The patients were selected according to the following criteria:

Patients having moderate to severe chronic periodontitis, ${ }^{(19)}$ associated with moderately poorly controlled type $2 \mathrm{DM}$ (HbA1c between
$8 \%-10 \%)^{(18)}$ and with nearly of the same duration of diabetes (about 5 years).

Patients age range was from 45-65 years old and they were non smokers and free from any other systemic diseases or diabetic complications. Moreover those reporting intake of any medications other than oral hypoglycemic drugs, or treated with insulin injection were excluded. They should not receive any surgical or non surgical periodontal treatment in the previous 12 months but with no evidence of periapical or periodontal abscess.

The selected patients were classified randomly into 2 groups as follow:

Group 1 (control): Comprised 10 patients (9 females and 1 male) who were subjected to non surgical periodontal management (phase I therapy).

Group 2 (test): Comprised 10 patients (9 females and 1 male) who received systemically administrated homeopathic remedy (Traumeel tablets) (Fig.2) adjunctive to phase I therapy with a dose of 1 tablet 3 times daily for 1 week.

\section{Materials:}

Traumeel drug is a homeopathic remedy which had been used in the present study(Biologische Heilmittel Heel GmbH company.Baden-Baden, Germany.www.heel.com). Traumeel s is composed of many botanical natural materials and minerals. ${ }^{(20)}$ such as:

Hamamelis, Echinacea, Aconitum napellus (Aconite), Hepar sulfuris Arnica (mountain arnica) which have anti-inflammatory, analgesic,antiviral, antibacterial, antioxidant effects and can also stimulate the healing of wounds.

\section{Methods}

The study was approved by the committee of ethics and an informed consent was obtained from each patient after providing detailed information and description of the study. 
All patients were subjected to the following steps:

\section{A) Clinical periodontal examination which included}

Probing pocket depth (PPD) (21), Clinical attachment loss (CAL) (21) and Modified Gingival index (MGI). ${ }^{(22)}$ of all the remaining teeth were measured at Six sites and the average was calculated before treatment and after 3 months of follow up.

\section{B) Laboratory investigations which included:}

Glycosalated hemoglobin, was used to evaluate if there is improvement in glycemic control, while Fasting blood glucose level and fasting blood insulin level were used to evaluate if the periodontal treatment in group one or two has a direct effect on blood glucose level and fasting blood insulin which are considered to be an indicator for improvement in insulin resistance. The Blood samples were taken after overnight fasting and the levels of the following compounds were measured at baseline and after 3 months for the two groups. Moreover the level of serum TNF- $\alpha$ was also assessed before treatment and after 3 months of follow up.

\section{C) Periodontal treatment which included:}

Non surgical periodontal management was in form of phase I therapy including: plaque control, full mouth supra and subgingival scaling, root planing and coronoplasty when needed.

\section{Statistical analysis:}

Data were fed to the computer and analyzed using IBM SPSS software package version 20.0. Student t-test was used to compare two groups for normally distributed quantitative variables. Paired t-test and ANOVA and Post Hoc test (LSD) was assessed for comparison between different periods.

Mann Whitney test was used to compare between two groups for abnormally distributed quantitative variables. Pearson coefficient was used to correlate between quantitative variables. Significance of the obtained results was judged at the $5 \%$ level.

\section{RESULTS}

A total of 20 patients having type 2 diabetes mellitus associated with periodontitis (2 males and 18 females) were evaluated in the present study. They were divided into two groups: group 1 (control) Comprised 10 patients 9 females and 1 male who were subjected to non surgical periodontal management in the form of phase I therapy while group 2 (test) Comprised 10 patients 9 females and 1 male who received systemically administrated Traumeel tablets.

Concerning the drug used in the present study, no adverse reactions were reported. The gingival tissues in all selected sites appeared healthy with no signs of inflammation.

Table 1 shows a comparison between the test and control group at base line and after a follow up period of 3 months regarding the main periodontal characteristics and lab investigations.

The levels of clinical periodontal variables including PPD, CAL, and MGI, were improved significantly in both groups test and control after 3 months (fig.1-4) (at $\mathrm{p} \leq 0.05)$ and on comparing those variables after 3 months the difference between the 2 groups was statistically significant ( at $\mathrm{p} \leq 0.05$ ).

Concerning FBG, FBI and glycosylated $\mathrm{Hb}$ (Gly.) all of them decreased significantly after treatment in both groups (at $\mathrm{p} \leq 0.05$ ). On comparing those variables after 3 months the difference between the 2 groups was statistically significant for FBI while for FBG and Glycosylated $\mathrm{Hb}$; it was not statistically significant( at $\mathrm{p} \leq 0.05$ ).

Regarding serum and crevicular TNF both of them decreased significantly after treatment in both groups (at $\mathrm{p} \leq 0.05$ ) and on comparing them after 3 months there was a statistically significant difference between the 2 groups (at $p \leq 0.05$ ).

On the other hand on comparing $\mathbf{D}$ between the 2 groups there was a statistically significant difference in all mentioned variables except crevicular TNF and Glycosylated $\mathrm{Hb}$. 
Moreover Table 2 shows the correlation between the clinical periodontal parameters including (CAL, PPD and MGI) and lab investigations including FBG and FBI .

The present study proved that there was a statistically significant positive correlation between
CAL and both FBG and FBI in test group( at $\mathrm{p} \leq$ 0.002). While regarding $\mathbf{D}$ which means difference between baseline and after 3 months there was a statistically significant positive correlation between all the clinical periodontal parameters and both FBG and FBI in test group only at $\mathrm{p} \leq 0.01$ for $\mathrm{CAL}$ and PPD and at $\mathrm{p} \leq 0.013$ for MGI.

TABLE (1) Comparison between the studied groups according to different parameters

\begin{tabular}{lcccccc}
\hline & \multicolumn{3}{c}{ Control group } & \multicolumn{3}{c}{ Test group } \\
\cline { 2 - 7 } & $\begin{array}{c}\text { Baseline } \\
(\mathbf{n = 1 0})\end{array}$ & $\begin{array}{c}\text { After 3 months } \\
(\mathbf{n = 1 0})\end{array}$ & $\begin{array}{c}\mathbf{D} \\
(\mathbf{n = 1 0})\end{array}$ & $\begin{array}{c}\text { Baseline } \\
(\mathbf{n}=\mathbf{1 0})\end{array}$ & $\begin{array}{c}\text { After 3 months } \\
(\mathbf{n = 1 0})\end{array}$ & $\begin{array}{c}\mathbf{D} \\
(\mathbf{n}=\mathbf{1 0})\end{array}$ \\
\hline Serum TNF & $12.15 \pm 6.26$ & $10.75 \pm 6.06^{\#}$ & $1.40 \pm 0.64$ & $8.67 \pm 2.10$ & $4.49^{*} \pm 0.88^{\#}$ & $4.18^{*} \pm 1.81$ \\
Crev. TNF & $7.60 \pm 2.34$ & $6.51 \pm 2.32^{\#}$ & $1.09 \pm 0.52$ & $2.99 \pm 1.39$ & $1.13^{*} \pm 0.77^{\#}$ & $1.85 \pm 1.21$ \\
GLy & $9.61 \pm 0.47$ & $8.45 \pm 0.51^{\#}$ & $1.16 \pm 0.44$ & $9.85 \pm 0.75$ & $7.88 \pm 0.86^{\#}$ & $1.97 \pm 1.16$ \\
PPD & $4.50 \pm 0.53$ & $3.0 \pm 0.82^{\#}$ & $1.50 \pm 0.71$ & $4.30 \pm 0.82$ & $1.50^{*} \pm 0.53^{\#}$ & $2.80^{*} \pm 0.92$ \\
CAL & $4.30 \pm 0.67$ & $2.80 \pm 0.79^{\#}$ & $1.50 \pm 0.71$ & $4.30 \pm 0.82$ & $1.50^{*} \pm 0.53^{\#}$ & $2.80^{*} \pm 0.92$ \\
MGI & $1.83 \pm 0.60$ & $0.80 \pm 0.26^{\#}$ & $1.03 \pm 0.53$ & $2.25 \pm 0.90$ & $0.35^{*} \pm 0.41^{\#}$ & $1.90^{*} \pm 1.02$ \\
FBI & $20.91 \pm 5.43$ & $19.31 \pm 4.87^{\#}$ & $1.60 \pm 0.93$ & $11.96 \pm 2.81$ & $8.57^{*} \pm 3.08^{\#}$ & $3.39^{*} \pm 1.31$ \\
FBG & $266.4 \pm 23.0$ & $248.5 \pm 28.8^{\#}$ & $17.9 \pm 6.9$ & $229.1 \pm 66.4$ & $184.7 \pm 88.8^{\#}$ & $44.4^{*} \pm 32.7$ \\
\hline
\end{tabular}

Data was expressed in mean $\pm S D$. \#: For comparing between Baseline and After 3 months at $p \leq 0.05$

*: For comparing between control and tablet after 3 months and $D$ at $p \leq 0.05 \quad$ D: Difference between baseline and after 3 months TNF, Tumor necrosis factor,Crev.TNF,Crevicular tumor necrosis factor, GLY. ,Glycosylated hemoglobin,PPD, Probing pocket depth,CAL, Clinical attachment loss,MGI, Modified Gingival index,FBI,Fasting blood insulin,FBG,Fasting blood glucose.

TABLE (2) Correlation between clinical periodontal variables, FBG and FBI

\begin{tabular}{|c|c|c|c|c|c|c|c|c|}
\hline & \multicolumn{4}{|c|}{ Control group } & \multicolumn{4}{|c|}{ Test group } \\
\hline & \multicolumn{2}{|c|}{ FBI } & \multicolumn{2}{|c|}{ FBG } & \multicolumn{2}{|c|}{ FBI } & \multicolumn{2}{|c|}{ FBG } \\
\hline & $\mathbf{R}$ & $\mathbf{P}$ & $\mathbf{r}$ & $\mathbf{P}$ & $\mathbf{R}$ & $\mathbf{P}$ & $\mathbf{r}$ & p \\
\hline \multicolumn{9}{|l|}{ Baseline } \\
\hline CAL & 0.236 & 0.512 & 0.134 & 0.711 & 0.044 & 0.904 & 0.093 & 0.798 \\
\hline PPD & -0.259 & 0.471 & 0.485 & 0.155 & 0.154 & 0.670 & -0.200 & 0.580 \\
\hline MGI & 0.256 & 0.475 & -0.456 & 0.186 & 0.289 & 0.418 & 0.197 & 0.585 \\
\hline \multicolumn{9}{|c|}{ After 3 months } \\
\hline CAL & -0.462 & 0.178 & 0.224 & 0.533 & $0.683^{*}$ & 0.030 & $0.844^{*}$ & 0.002 \\
\hline PPD & -0.092 & 0.800 & 0.104 & 0.775 & 0.355 & 0.315 & 0.483 & 0.157 \\
\hline MGI & 0.298 & 0.403 & -0.624 & 0.054 & 0.165 & 0.648 & -0.263 & 0.463 \\
\hline \multicolumn{9}{|l|}{ D } \\
\hline CAL & -0.418 & 0.230 & -0.329 & 0.354 & $0.860^{*}$ & 0.001 & $0.912^{*}$ & $<0.001$ \\
\hline PPD & -0.418 & 0.230 & -0.329 & 0.354 & $0.860^{*}$ & 0.001 & $0.912^{*}$ & $<0.001$ \\
\hline MGI & 0.166 & 0.646 & 0.594 & 0.070 & $0.937^{*}$ & $<0.001$ & $0.748^{*}$ & 0.013 \\
\hline
\end{tabular}




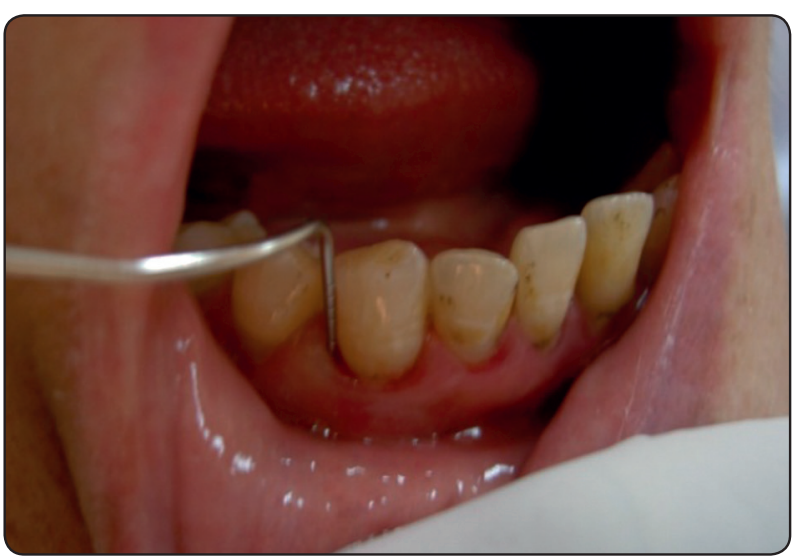

Fig. (1) Preoperative CAL in control group

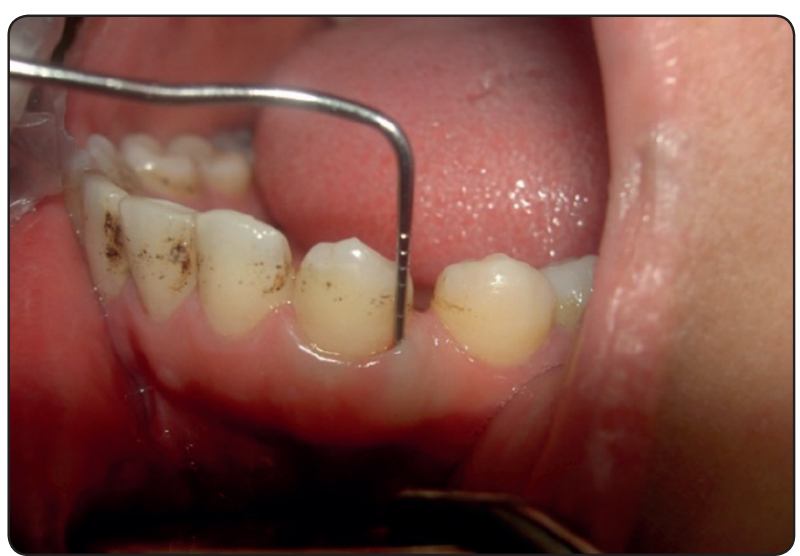

Fig. (3) Preoperative CAL in test group

\section{DISCUSSION}

Diabetes mellitus is a metabolic diseases which characterized by hyperglycemia resulting from defect in insulin secretion, action or both. ${ }^{(23)}$ It is often associated with an unacceptably high disease burden especially in developing countries. In Egypt it is considered a major clinical and public health problem. ${ }^{(24)}$

Periodontal disease is a chronic oral infectious disease and considered as the sixth complication of diabetes. Both diseases are of high incidence and the incidence of diabetes associated with periodontal disease has an upward trend among old people. ${ }^{(23)}$

Evidence has consistently indicated that diabetes

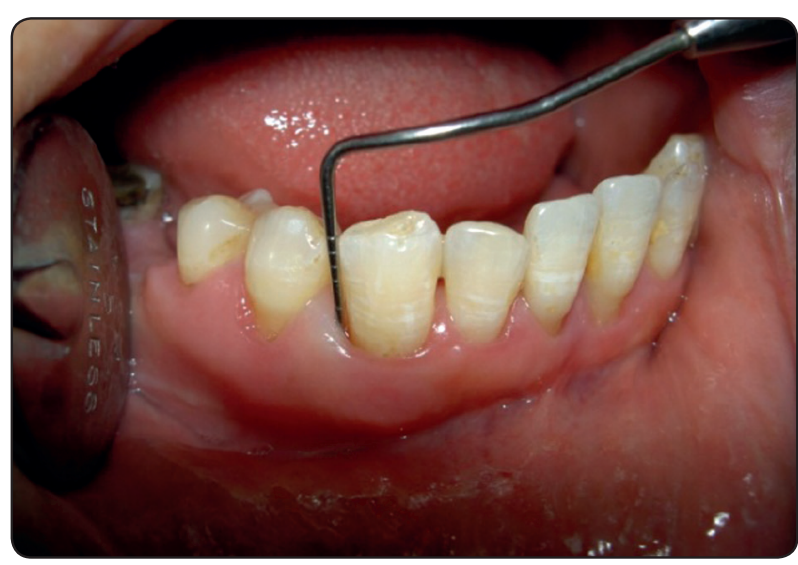

Fig. (2) CAL after 3 months in control group

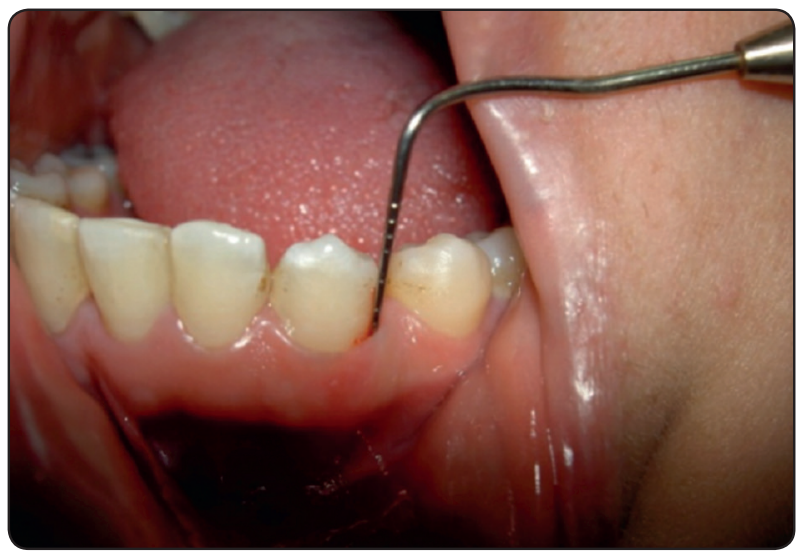

Fig. (4) CAL after 3 months in test group

is a risk factor for increased severity of gingivitis and periodontitis.(25,26) Conversely, periodontitis may be a risk factor for worsening glycemic control among patients with diabetes and may increase the risk of diabetic complications. ${ }^{(26,27)}$ Thus the relation between periodontal health and diabetes has been described as bidirectional. ${ }^{(28)}$

Traumeel is a homeopathic remedy that represent a good example of bioregulatory medicine through modulating inflammatory pathways by downregulating proinflammatory cytokines and up regulating anti-inflammatory cytokines, ${ }^{(29-31)}$ More over Žilinskas J etal (2011) ${ }^{(17)}$ proved that traumeel had an antioxidant effect thus using traumeel with its antioxidant and anti-inflammatory properties 
was very beneficial for periodontitis treatment and so improving patients Glycemic control, FBG and FBI according to the results of this study.

In the present study, we noted that PPD, CAL, and MGI were significantly decreased in the test group and control group after 3 months as well as the HbAlc, serum and crevicular TNF- $\alpha$, concerning FBG and FBI levels they also decreased and are a good indication for the improvement of insulin resistance as when the FBI decrease this means that more insulin start to enter the cells to start to consume it for energy production which occur only when insulin resistance decrease.

These results confirmed our hypothesis that periodontal treatment not only reduces clinically evident inflammation, but also improves the glycemic control and reduces insulin resistance (IR). These findings indicate that inflammation is involved in the pathogenesis of both DM and periodontitis.

Moreover on comparing $\mathbf{D}$ between the 2 groups there was a statistically significant difference in all mentioned variables except crevicular TNF and Glycosylated $\mathrm{Hb}$ which proves that traumeel drug when used adjunctive to conventional periodontal treatment had provided more reduction in both clinical and laboratory variables than periodontal treatment alone due to its anti-inflammatory and antioxidant effect.

This study also proved the presence of a statistically significant positive correlation between the $\mathbf{D}$ of FBG and FBI as an indicator for insulin resistance and the $\mathbf{D}$ of periodontal findings including CAL,PPD and MGI in traumeel drug group which confirm the study hypothesis about the positive impact of adding traumeel drug as an antioxidant and anti-inflammatory drug to the treatment protocol for type $2 \mathrm{DM}$ having chronic periodontitis. Thus traumeel drug in a conjunction to phase 1 periodontal therapy had led to both improvement in periodontal health and improvement in patients FBG and FBI due to reduction in IR.
FBG and FBI are considered as an indicator for insulin resistance because it is well known that IR is usually evaluated by the HOMA index (homoeostasis model assessment of the IR index, HOMA-IR), that depend on the values of FBG and FBI and so reduction in both results in reduction in IR. ${ }^{(18)}$

There is an agreement between our results and those obtained by OU Long etal $(2011)^{(32)}$ who investigate the effect of periodontal treatment on levels of blood glucose (Glu) and glycosylated hemoglobin (HbA1c) among elderly patients with type 2 diabetes and periodontal disease. They used anti-inflammatory drugs, such as iodine glycerin or Periocline placed into periodontal pocket adjunctive to subgingival scaling, root planing and removal of infected tissue and occlusal adjustment. They found that periodontal treatment can effectively reduce the level of Glu and HbA1c as well as improve the periodontal condition by reducing probing depth (PD), attachment loss (AL) significantly, in elderly type-2 diabetes patients with periodontal disease.

However, Jones et al indicated that periodontal therapy has no statistically significant effect on glycemic control ${ }^{(33)}$. This could be attributed to considerable differences in methodology, sample sizes and composition of the groups included in the studies.

More over our results are also close to that reached by Sun WL etal $(2011)^{(18)}$ who conducted a study to evaluate the effects of periodontal intervention in form of scaling and root planing on inflammatory cytokines, insulin resistance (IR), and metabolic control ( $\mathrm{HbA1c})$ and to investigate the relationship between type 2 diabetes mellitus (T2DM) with moderately to poor glycemic control and chronic periodontitis. They concluded that Periodontal intervention can improve glycemic control through reducing $\mathrm{HbA} 1 \mathrm{c}$, lipid profile, IR, serum inflammatory cytokine levels specially IL-6 and TNF- $\alpha$ in moderately poorly controlled T2DM patients. 


\section{CONCLUSION}

Traumeel drug proved to have no adverse effects on patients with type $2 \mathrm{DM}$ associated with chronic periodontitis during the entire study period (3months). The adjunctive use of homeopathic traumeel tablets with periodontal intervention offered better results concerning periodontal and metabolic state. Moreover the reduction in levels of TNF- $\alpha$ in serum was associated with reduction in FBS and FBI which indicates the reduction in insulin resistance and consequently $\mathrm{HbA} 1 \mathrm{c}$ in type 2 DM patients.

\section{REFERENCES}

1- Mealey BL, Rose LF. Diabetes mellitus and inflammatory periodontal diseases. Curr Opin Endocrinol Diabetes Obes. 2008; 15: 135-41.

2- Brain L, Mealy S,Gloria L, Ocampo. Diabetes mellitus and periodontal disease. periodontology 2000. 2007; 44:127-53

3- Bergman RN,Ader M.Free fatty acids and pathogensis of type 2 diabetes mellitus. Trends Endocrinal Metab. 2000;11:351-6.

4- Taylor GW, Borgnakke WS. Periodontal disease: associations with diabetes, glycemic control and complications. Oral Dis. 2008;14: 191-203.

5- Mealey BL, Rose LF. Diabetes mellitus and inflammatory periodontal diseases. Curr Opin Endocrinol Diabetes Obes. 2008; 15: 135-41.

6- Garlet GP, Cardoso CR, Campanelli AP, Ferreira BR, Avila-Campos MJ, Cunha FQ, Silva JS, The dual role of p55 tumour necrosis factor-alpha receptor in Actinobacillus actinomycetemcomitans-induced experimental periodontitis: host protection and tissue destruction. Clin Exp Immunol. 2007; 147(1):128-38.

7- Salvi GE, Carollo-Bittel B, Lang NP. Effects of diabetes mellitus on periodontal and peri-implant conditions: update on associations and risks. J Clin Periodontol. 2008; 35: 398-409.

8- Nishimura F, Iwamoto Y, Mineshiba J, Shimizu A, Soga Y, Murayama Y. Periodontal disease and diabetes mellitus: The role of tumor necrosis factor-alpha in a 2-way relationship. J Periodontol. 2003;74(1):97-102.
9- Padeh B. The Effect of Periodontal Treatment on the Level of Free Radicals in the Saliva department of oral and maxillofacial surgery, Medical CenterTieberia.ClinicalTrials. gov on July 15, 2010.

10- Mohamed AK, Bierhaus. A, Schiekofer. S, Tritscler. H, Ziegler. R, Nawroth. P.P, The role of oxidative stress and NK-Kappa B activation in late diabetic complications, Biofactors. 1999;10:157-67.

11- Aragno ME, Tamagno V, Gato E, Brignardello, S, Parola O, Danni G. Boccuzzi, Dehydroepiandrosterone protects tissues of streptozotocin-treated rats against oxidative stress. Free Radic. Biol. Med. 1999; 26(11/12): 1467-147

12- Bonnefont Rousselot, D., J.P. Bastard, M.C. Jaudon, J. Delattre, Consequences of the diabetic status on the oxidant/ antioxidant balance, Diabetes Metab. 2000;26: 163-76.

13- Robertson, R.P., Chronic oxidative stress as a central mechanism for glucose toxicity in pancreatic islet beta cells in diabetes, J. Biol. Chem. 2004; 279(41):42351-4.

14- Ballard R. Homeopathy: An overview. Australian Family Physician. 2000;29(12):1145-1148.

15- Porozova S, Cahalon L, Weiser M, Branski D, Lider O and Oberbaum M.Inhibition of IL-1b and TNF-a Secretion from Resting and Activated Human Immunocytes by the Homeopathic Medication Traumeel. Clinical \& Developmental Immunology. 2004; 11 (2): 143-9.

16- Ludmila G, Nina S, Pavel G, Nadejda D, Leonid LC. Treatment of patients with parodontitis by antihomotoxic pharmacotherapy with Traumeel S . OHDMBSC. 2004; vol III:4.

17- Zilinskas J, Zekonis J, Zekonis G, Sadzevičienė R, Sapragonienė M, Navickaitė J, Barzdžiukaitė I. Med Sci Monit. Inhibition of peripheral blood neutrophil oxidative burst in periodontitis patients with a homeopathic medication Traumeel S. Stomatologija Baltic Dental and Maxillofacial Journal. 2011; 2;17(5):CR284-91.

18- Sun WL, Chen LL, Zhang SZ, Wu YM, Ren YZ, QinGM. Inflammatory Cytokines, Adiponectin, Insulin Resistance and Metabolic Control after Periodontal Intervention $n$ Patients with Type 2 Diabetes and Chronic Periodontitis. Intern Med 2011; 50: 1569-74.

19- Armitage GC. Development of a classification. System for periodontal diseases and conditions. Ann Periodontol 1999; 4: 1-6

20- Müller-löbnitz C, Göthel D. Review of the clinical efficacy of the multicomponent combination medication traumeel and its components. Alter Ther Health Med 2011; 17: 18-31. 
21- Glavind L, Loe H. Error in the clinical assessment of periodontal destruction. J Periodont Res 1967; 2: 180-4.

22- Lobene RR, Weatherford T, Ross NM. Modified gingival index. Clin Prev Dent 1986; 8: 3-6.

23- American diabetes association. Diagnosis and classification of diabetes. Diabetes care 2013; 36: 567-74.

24- Arafa NAS, Essam GE, Amin D. The Epidemiology of Diabetes Mellitus in Egypt: Results of a National Survey. Egy J Community Med 2010; 28: 3.

25- Gerard J. Linden, Amy Lyons, Frank A. Scannapieco. Periodontal systemic associations: review of the evidence. J Periodontol 2013; 84:4-s, Pages S8-S19.

26- John J. Taylor1, Philip M. Preshaw1 and Evanthia Lalla2 A review of the evidence for pathogenic mechanisms that may link periodontitis and diabetes. J Periodontol 2013; 84:4-s, Pages S113-S34.

27- Borgnakke WS, Ylöstalo PV, Taylor GW, Genco RJ. Effect of periodontal disease on diabetes: systematic review of epidemiologic observational evidence. J Periodontol 2013; 84: S135-52.

28- Chapple IL, Genco R. Diabetes and periodontal diseases: consensus report of the Joint EFP/AAP Workshop on
Periodontitis and Systemic Diseases. J Periodontol 2013; 84: S106-12.

29- Heine H, Schmolz M. immunoregulation via 'bystander suppression' needs minute amounts of substances - a basis for homeopathic therapy? Med Hypotheses 2000; 54: 392-3.

30- Conforti A, bertani S, Chirumbolo S, lussignoli S, bellavite P, Metelmann h. experimental studies on the anti-inflammatory activity of a homeopathic preparation. Biomed Ther 1997; 15: 28-31.

31- Conforti A, Bellavite P, Bertani S, Chiarotti F, Menniti-Ippolito F, Raschetti R. rat models of acute inflammation: a randomized controlled study on the effects of homeopathic remedies. BMC Complement Altern Med 2007; 7: 1.

32- OU Long and LI Ru-fan . Effect of periodontal treatment on glycosylated hemoglobin level in elderly patients with periodontal disease and type 2 diabetes. Chin Med J 2011;124(19):3070-3073.

33- Jones JA, Miller DR, Wehler CJ, et al. Does periodontal care improve glycemic control? The Department of Veterans Affairs Dental Diabetes Study. J Clin Periodontol 2007;34: 46-52. 